\title{
Innovación y creatividad como motores de desarrollo y RSC. El caso de las empresas de Guayaquil (Ecuador)
}

\author{
José Manuel Saiz Álvarez \\ Marlene Mariluz Mendoza Macías
}

\section{RESUMEN}

La implantación de estrategias corporativas basadas en RSC está en un momento inicial en las empresas ecuatorianas. A lo largo de este trabajo se desarrolla el impacto que tendría una mayor implantación de dicha política en los planes estratégicos de las empresas, para lo cual los autores han realizado una encuesta a 70 empresarios de industrias estratégicas de la ciudad de Guayaquil. Para mejorar la implantación de la RSC en la empresa ecuatoriana, y en concreto la situada en Guayaquil, primera ciudad de Ecuador en población y primera en tráfico portuario, los autores proponen un nuevo modelo de gestión basado en responsabilidad social para que los beneficios derivados de dicha implantación puedan mejorar las condiciones de vida, no solamente de los profesionales que trabajan en las empresas, sino también de la población en su conjunto.

PALABRAS CLAVE: Responsabilidad social corporativa, desarrollo sostenible, innovación estratégica, stakeholder, creatividad.

CLAVES ECONLIT: M140, M100, G320.

Cómo citar este artículo: SAIZ, J.M. \& MENDOZA, M.M. (2015): "Innovación y creatividad como motores de desarrollo y RSC. El caso de las empresas de Guayaquil (Ecuador)", CIRIEC-España, Revista de Economía Pública, Social y Cooperativa, 85, 33-53.

Correspondencia: José Manuel Saiz Álvarez, TEC de Monterrey (México) y Universidad Nebrija (España); Marlene Mariluz Mendoza Macías, Universidad Católica de Santiago de Guayaquil (Ecuador). E-mail de contacto: josemanuelsaizalvarez@gmail.com. 


\section{Innovation et créativité en tant que moteurs de développement et RSE. Le cas des entreprises de Guayaquil (Équateur)}

RÉSUMÉ : La mise en œuvre des stratégies d'entreprise fondées sur la RSE se situe à un stade précoce dans les entreprises équatoriennes. Ce travail développe l'impact qu'obtiendrait une plus grande mise en œuvre de cette politique dans les plans stratégiques des entreprises. Dans cette optique, les auteurs ont mené une enquête auprès de 70 entrepreneurs dans les industries stratégiques de la ville de Guayaquil. Afin d'améliorer la mise en œuvre de la RSE au sein du secteur entrepreneurial équatorien, et en particulier celui situé à Guayaquil, la première ville équatorienne en termes de population et de trafic portuaire, les auteurs proposent un nouveau modèle de gestion basé sur la responsabilité sociale de sorte que les avantages de cette mise en œuvre puissent améliorer les conditions de vie, non seulement des professionnels travaillant dans les entreprises, mais aussi celles de la population dans son ensemble.

MOTS CLÉ : Responsabilité sociale des entreprises, développement durable, innovation stratégique, stakeholder, créativité.

\section{Innovation and creativity as drivers of development and CSR. A case study of companies in Guayaquil (Ecuador)}

ABSTRACT: The implementation of corporate strategies based on CSR is at an early stage in Ecuadorian companies. This paper explains the impact that greater implementation of CSR policy would have on the strategic plans of companies. To this end, the authors conducted a survey of 70 entrepreneurs working in strategic industries in the city of Guayaquil. To improve CSR implementation in Ecuadorian companies, specifically those located in Guayaquil (Ecuador's most populous city and busiest port), the authors propose a new management model based on social responsibility. The benefits of its implementation could improve not only the living conditions of professionals working in companies, but also those of the population as a whole.

KEY WORDS: Corporate Social Responsibility, sustainable development, strategic innovation, stakeholder, creativity. 


\section{1.- Introducción}

En la actualidad, la innovación forma parte de la Responsabilidad Social Corporativa (en adelante, $\mathrm{RSC}$ ), aunque, salvo excepciones, no con la importancia con la que debería ser considerada. Para ser líder en su sector de actividad, una empresa socialmente responsable debe ser una empresa con afán de mejora continua, a partir de la innovación, con independencia de que exista o no competencia. Como resultado de este hecho, la implementación de estrategias innovadoras crece de manera importante en las empresas que buscan ser sostenibles por factores tales como: el carácter "glocal" de las estrategias empresariales como indicador que la organización piensa globalmente y actúa localmente, la mayor competencia existente en el comercio internacional con la llegada de nuevos actores económicos al mercado, la existencia de un flujo continuo de l+D+i que genera constantes mejoras tecnológicas, y el cada vez más reducido deterioro medioambiental.

Ante un contexto cambiante, las presiones que preocupan a los emprendedores son de índole económica, social y ambiental, por lo que su gestión tiende a la búsqueda del equilibrio y el desarrollo armonioso de estos tres componentes para lograr el éxito sostenible de su empresa. La RSC aparece así como una respuesta estratégica a las diversas exigencias llevadas a cabo por los stakeholders hacia la organización. En este contexto, ¿la innovación y la creatividad constituyen modelos de crecimiento que favorecen, a su vez, la realización de políticas basadas en RSC?. Para responder a esta cuestión, comenzaremos analizando la importancia de la RSC para a continuación estudiar los beneficios de su aplicación, incidiendo de forma especial en la relación entre la RSC y el talento humano, la creatividad y el medioambiente, lo que sentará las bases para analizar la implantación de dicha estrategia en las empresas de Guayaquil. Aplicación que se examina para 70 gerentes guayaquileños. El trabajo finaliza con unas conclusiones en donde se dan unas perspectivas de futuro.

\section{2.- La importancia de la RSC}

Es importante destacar que no hay una definición de la RSC que sea comúnmente aceptada, por las diferentes perspectivas existentes en la literatura económica sobre la forma de concebirla. En la década de los años 1960 y 1970 se priorizaba la postura neoclásica que favorecía a los intereses de los empresarios, al afirmar que una empresa era socialmente responsable si era capaz de aumentar 
los dividendos y los beneficios percibidos por los accionistas, además de generar empleo privado (Friedman, 1962 y 1970).

Esta perspectiva tan focalizada abrió su visión en la década de 1980, cuando la RSC deja de tener una visión meramente empresarial parece hacerse más humanista y filantrópica, visión que hasta la actualidad se mantiene en algunas organizaciones. Este enfoque humanista de la RSC hace referencia a una forma de atención de los intereses de la sociedad a través de donaciones, regalos y ayudas sociales hacia los más necesitados. De allí surgen una serie de formas de practicar la filantropía desde las empresas hacia la sociedad, por lo que desde dicha óptica son socialmente responsables aquellas organizaciones que más acciones de filantropía realizan. En algunos casos, y una vez conseguidos los objetivos empresariales, parte de la actividad se dirige hacia la generación de una imagen corporativa centrada en la bondad y la preocupación respecto de los problemas sociales.

En la década de 1990 aparece una versión más amplia, pues ya se consideran tanto los mayores beneficios para los empresarios, como también se tienen en cuenta las acciones de filantropía que muestran una actuación socialmente responsable. Esto hace que se logre la RSC cuando se equilibran los intereses de los stakeholders en la empresa (Freeman, 1988 y 1994)(Guildford, 1995)(Mitchell, 1997)(Carroll, 1999). Esta forma de concebir la RSC obliga a dar un giro importante en la forma de actuación de las organizaciones. La sociedad, el ambiente y los involucrados en la empresa pasan a ser tan importantes como los dueños. Si uno de ellos no es adecuadamente atendido se corre el riesgo de minimizar la posición competitiva de la organización, así como mermar su producto y/o servicio de marca.

Hoy son muy relevantes las acciones de RSC integradas que se realizan en torno a los intereses de los stakeholders. Desde dicho enfoque, y según Vaca, Moreno y Riquel (2007), la RSC implica que "los directivos deben ir más allá de la mera satisfacción de los accionistas o propietarios, orientándose al establecimiento de relaciones equilibradas con todos sus stakeholders" (p. 3139), lo que involucra comprender el papel de cada agente en la sociedad, sus derechos y responsabilidades. Como resultado, el concepto de RSC está adquiriendo cada vez más relevancia en el mundo actual debido al buen uso de las Tecnologías de la Información y la Comunicación (TIC)(Porter, 1999).

Dentro de este contexto más amplio, la RSC es la capacidad de respuesta que tiene cualquier organización frente a las consecuencias de sus actividades sobre los diferentes grupos con los que se relaciona. Los fundamentos sobre los que se asienta el paradigma de empresa responsable y sostenible son, de acuerdo a Olcese, Rodríguez y Alfaro (2008): "la apertura y la sensibilidad hacia el entorno, el sentido de comunidad, la capacidad innovadora, la consideración de largo plazo y la creación de valor" (p. 20). Una creación de valor que mejora, a su vez, la reputación corporativa y de marca, así como fideliza al capital intelectual, como suma de capital humano, capital relacional y capital estructural (Saiz-Alvarez, 2014), existente en la organización. 
Por otra parte, no hay oficialidad sobre la forma de medir la RSC. Han surgido informes con indicadores diversos que intentan medir el grado de RSC en las organizaciones, tales como el GRI (Global Reporting Initiative), la Iniciativa del Pacto Mundial y los indicadores de Ethos, pero el común denominador a destacar es la carencia de comparación entre ellos. Se bien el GRI es uno de los indicadores que tiene ahora mayor aceptación, no es el único ni el definitivo. Como aún no hay una estandarización, continúan las investigaciones para desarrollar un instrumento de medición que permita comparar los indicadores de la responsabilidad social en las empresas.

A fines del 2010 se emitieron las normas ISO 26000 como una guía a las organizaciones que voluntariamente deseen implementar acciones socialmente responsables. En el Ecuador existe el CERES (Consorcio Ecuatoriano para la Responsabilidad Social), en la que están asociadas las empresas ecuatorianas que buscan obtener una posición pionera en la aplicación de la RSC. En el año 2011 el CERES se comprometió con los objetivos del Pacto Global, lo cual es un avance importante por parte de las empresas ecuatorianas. También en Ecuador se promueve la actuación con responsabilidad social en las empresas desde el desarrollo de su actividad productiva, tarea encabezada por el Ministerio de industrias y productividad. Se reconoce con los sellos "Hace bien" y "Hace mejor" a las empresas comprometidas con las cuatro éticas: trabajadores, comunidad, medio ambiente y Estado.

Según la Norma ISO 26000 (2010), la característica esencial de la RSC:

"Es la voluntad de las organizaciones de incorporar consideraciones sociales y ambientales en su toma de decisiones y de rendir cuentas por los impactos de sus decisiones y actividades en la sociedad y el medio ambiente. Esto implica un comportamiento transparente y ético que contribuya al desarrollo sostenible, cumpla con la legislación aplicable y sea coherente con la normativa internacional de comportamiento. También implica que responsabilidad social esté integrada en toda la organización, se lleve a la práctica en sus relaciones y tenga en cuenta los intereses de las partes" (p. 7)

Los clientes exigen productos de la máxima calidad, considerando de forma preferente a los productos que generen bienestar a las personas y que no generen externalidades negativas en el medio ambiente. Esto se refleja en la creación de los movimientos de defensa al consumidor que son "un movimiento social que pretende compensar el poder y los derechos de los compradores" (Schwalb, 2011, p. 95) en Peinado (2011) frente a unos vendedores que suelen estar en coalición, ya sea para formar grupos empresariales, cooperativas y comunidades de bienes. Paralelamente, la sociedad demanda un mayor cuidado al medio ambiente, lo que lleva a que se establezca el cumplimiento de derechos de las personas, el derecho a la vida y a la satisfacción de sus necesidades básicas, los derechos laborales y la protección ambiental, entre otros. Como la realidad de las organizaciones no está aislada de los demás agentes con los que se interrelaciona, el empresario tiene claro su desafío de crecimiento y desarrollo considerando atender intereses que tradicionalmente han sido diferidos y/o descuidados porque "los ejecutivos reconocen que el crecimiento económico de largo plazo ya no es posible a menos que ese crecimiento sea social y ambientalmente sostenible" (Epstein, 2009, p. 3). 
Por otra parte, bajo un contexto de inestable crecimiento, alta desigualdad, e importantes niveles de pobreza, la participación de las empresas es fundamental para lograr el cambio a favor del crecimiento y desarrollo que a la vez beneficien a sus negocios (Porter y Kramer, 2006)(Porter, 2011). La evidencia muestra aún una importante inequidad económica y social en los países de la región latinoamericana, lo que impide alcanzar un auténtico desarrollo económico que sea justo para toda la sociedad. Así, en el caso de Ecuador, el Índice de Desarrollo Humano para 2013 fue de 0,711, lo que lleva al Ecuador ocupar el puesto 98 del mundo. El coeficiente de Gini muestra la inequidad en la distribución de los ingresos, al 2013 el coeficiente de Gini fue de 0,485 para Ecuador y en marzo del 2014 se ubicó en 0,4601 (INEC, 2014). Si bien ha existido una ligera mejora en las cifras, no es suficiente para superar la inequidad existente en la república.

Así, son variados los frentes en los que deben actuar las empresas innovando en sus estrategias para destacar y/o sobrevivir en el referido contexto. "A menudo son sustanciales las consecuencias para los negocios cuando no consideran de manera efectiva los impactos que sus actividades tienen en la sociedad" (Epstein, 2009, p. xxiv). La actuación socialmente responsable es fundamental en las empresas a fin de obtener la licencia social para operar y favorecer su imagen. De acuerdo a Peinado (2011, p. 10) la RSC es "imprescindible por la necesidad para el sector privado de contribuir al desarrollo sostenible y más equitativo, en la lucha contra la desigualdad y a favor de la inclusión económica". En el mismo sentido se expresan Olcese, Rodríguez y Alfaro (2008, p. 29), ya que "la empresa es, sin duda, una de las instituciones sociales más importantes y con un mayor poder de influir, positiva o no tan positivamente, en el sistema económico, natural y social. En este sentido no cabe duda de que sobre la empresa recae parte de la responsabilidad de la situación actual de dicho sistema". Como resultado, las empresas tienen la oportunidad de actuar estratégicamente aportando al desarrollo del país y, a la vez, beneficiarse en sus negocios a partir de las innovaciones con un enfoque de RSC (Informe Forética, 2011).

Actuar en la línea de la RSC significa desarrollar un negocio generando beneficios para todos los involucrados, tanto empresarios como grupos de interés, lo que significa en términos más específicos aplicar prácticas laborales justas y crear relaciones sostenibles entre empleador-empleados, proveedor y clientes, gobierno, sociedad y cuidado del medio ambiente que aporten al bienestar de toda la sociedad y no solo de la empresa. Desde esta perspectiva se busca identificar las actuaciones basadas en la política de gestión estratégica que implementan las empresas de Guayaquil en torno a la RSC con sus stakeholders.

En la consecución del desarrollo sostenible, todos los agentes tienen derechos y responsabilidades y un papel importante de acción, pero sin duda el de mayor relevancia recae sobre los empresarios quienes deben transformar a sus empresas en un buen ciudadano corporativo. Ellas deben potenciar el adecuado ejercicio de la Responsabilidad Social. 


\section{Figura 1. Modelo de Gestión con RSC}

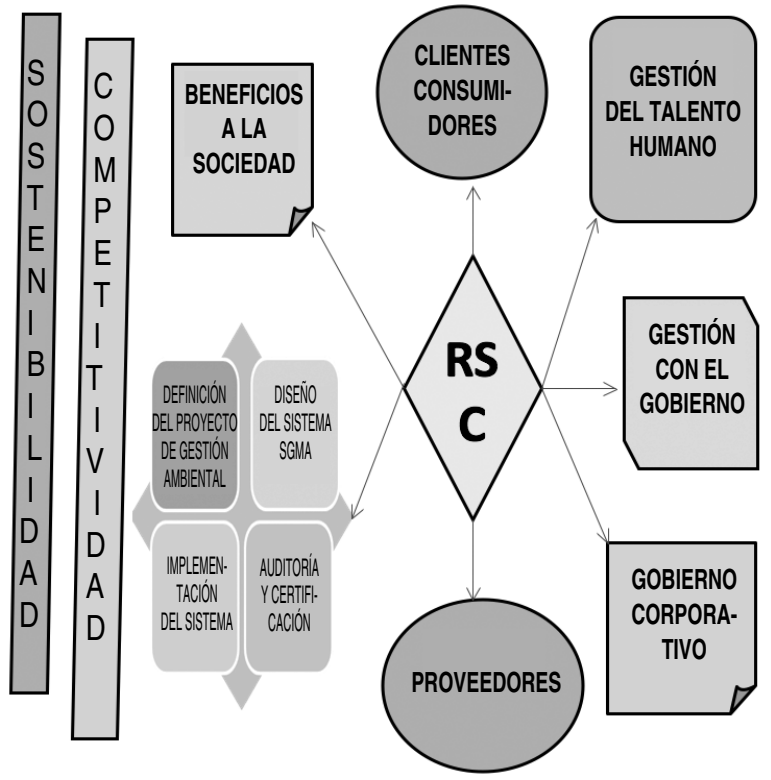

FUENTE: Elaboración propia.

En la figura 1 se muestra un esquema de aspectos teóricos de RSC vinculados al enfoque de los stakeholders. Aquí se identifica la participación general de las empresas en torno a la RSC como parte integral de un nuevo modelo de negocio que destaca estrategias innovadoras. En dicho esquema los objetivos de la empresa no son solo particulares, sino también de sus stakeholders y busca aportar al crecimiento y desarrollo del país donde los beneficios también son sociales porque "la RSC no es sólo rentable, es necesaria para mejorar la competitividad de las empresas y contribuir a mejorar la vida de muchas personas" (Peinado, 2011, p. 80).

\subsection{Beneficios de la RSC}

De acuerdo a Peinado (2011):

"La RSC es una versión más elaborada que forma parte de las actividades empresariales y tiene en cuenta las consecuencias sobre las distintas partes interesadas. Se incorporan asuntos sociales y ambientales en la estrategia empresarial de forma integrada. A través de estas prácticas responsables, llevadas a cabo como un elemento más de gestión de la empresa, se pueden conseguir mejoras competitivas que dan lugar a mayores beneficios. Al mismo tiempo, se contribuye a influir sobre las condiciones del entorno (al contribuir a una sociedad mejor y mayor estabilidad) que a su vez repercute positivamente en las operaciones" (p. 68). 
Es importante destacar la esencia del valor compartido al que se refieren Porter y Kramer (2006, p. 81) quienes indican que se debe actuar integradamente. Las empresas tienen una gran oportunidad al modificar sus estrategias de negocio considerando factores no observables que eran dejados en manos del sector público. De hecho, las empresas pueden realizar grandes actuaciones sociales y generar beneficios múltiples porque:

Las corporaciones exitosas necesitan una sociedad sana. La educación, los servicios de salud y la igualdad de oportunidades son esenciales para una fuerza laboral productiva [...]. En última instancia, una sociedad sana crea una demanda creciente para la empresa, al satisfacerse más necesidades humanas y crecer las aspiraciones. Cualquier negocio que persiga sus fines a expensas de la sociedad en la que opera descubrirá que su éxito es ilusorio $y$, en definitiva, temporal. Al mismo tiempo, una sociedad sana necesita de empresas exitosas. Ningún programa social puede competir con el sector de negocios a la hora de crear trabajos, riqueza e innovación que mejore los niveles de vida a través del tiempo.

La RSC genera beneficios que contribuyen al incremento en el valor de la empresa, además de aumentar su productividad, la fidelidad de sus clientes, las relaciones estables con sus proveedores, el compromiso de su talento humano, el apoyo del gobierno, la aceptación de la sociedad, la imagen positiva en el mercado y la mayor participación en el mismo, entre otros, según Forética (2011).

La difusión de la RSC es posterior a la modelización de la innovación y ambas crean un círculo virtuoso a medida que van madurando dentro del mismo (MacGregor y Fontrodona, 2008). De hecho, la innovación permite acelerar la puesta en marcha de políticas basadas en RSC por los buenos resultados empresariales conseguidos derivados del incremento de la producción y la reducción de costes, lo que repercute positivamente en la generación de EBITDA (Earnings before Interests, Taxes, Depreciation and Amortization). De hecho, las organizaciones, y en especial las Pequeñas y Medianas Empresas (PYME), generan una muy fuerte innovación cuando ponen en marcha políticas basadas en RSC (Bocquet y Mothe, 2011) como resultado de la atracción de nuevos clientes y stakeholders, lo que permite a su vez aumentar los nichos de mercado.

Según Perrine (2013), la RSC obliga a muchos cambios estructurales a las compañías en donde la innovación juega un papel fundamental. Uno de los cambios estructurales fundamentales que se han de llevar a cabo es la mejora de la comunicación, tanto en un sentido horizontal entre departamentos, como vertical, con un constante flujo de información entre la base y el ápice estratégico. Información caracterizada por ser transparente, accesible en tiempo real y constante en el tiempo. Cuando fallan estos flujos de información, la organización genera ineficiencias $\mathrm{X}$ que lleva a cuellos de botella y, en caso de persistir en el tiempo, al colapso de la organización.

Para lograr una mayor competitividad, el núcleo duro (core) estratégico debe estar caracterizado por una innovación que no debe entenderse solo en el campo tecnológico (innovación técnica), sino en todos los ámbitos (innovación económica). Una empresa puede innovar en las formas de tomar deci- 
siones, en sus estructuras organizacionales, en las estrategias para relacionarse con los clientes y proveedores, en el desarrollo del negocio, en los mecanismos de ventas y en el marketing de sus productos. Por ello, no solamente se trata de nuevas inversiones en maquinaria y equipos, sino que busca aportar al desarrollo de recursos y capacidades de la empresa a través de la innovación para generar mayor valor y a su vez beneficios, tanto a la sociedad en general como a la empresa en particular.

Como resultado, para ser una empresa innovadora exitosa requiere crear una cultura de continuos cambios en todas sus áreas, con un compromiso con todos los involucrados en la organización, y con un grado suficiente flexibilidad horizontal y vertical, así como de una adecuada capacidad de ajuste, apertura, comunicación y participación para poder desarrollar las ideas con éxito. En realidad, la empresa es como un ser vivo (visión organicista) en la que puede lograr mayor valor agregado a partir de la puesta en práctica de nuevas ideas. Así, las que desean ser exitosas en un entorno dinámico y pretenda disponer de unas mayores exigencias sociales, deberán seguir innovando considerando los intereses de sus stakeholders. En definitiva, deberán atreverse y arriesgarse a realizar acciones que conviertan a sus empresas en pioneras en los mercados.

\subsection{RSC y talento humano}

Desde la perspectiva de la actuación de la RSC, el talento humano se destaca como el agente más importante dentro de las corporaciones. Para Pelekais y Aguirre (2008) "la responsabilidad social tiene su fundamento en el interior de la organización, la cual asume los valores y principios que la regulan dentro de su plataforma filosófica, y es a través de la internalización de estos que se exterioriza hacia el entorno en el cual se encuentra asentada" (p. 19). De allí que, la atención a los intereses de los colaboradores refleja los valores al interior de la organización.

En la gestión del talento humano se debe estar vigilante para brindar un adecuado ambiente laboral, disponer de información transparente, y una motivación para crear medios para desarrollar colaboradores. Las acciones de motivación son múltiples, ya que parten de la compresión de las necesidades del trabajador, para continuar con los incentivos para el desarrollo de sus habilidades y el alcance de sus metas personales, capacitación, información e integración en el proceso de crecimiento y desarrollo de la organización. El aporte de la compañía al desarrollo de sus colaboradores tiene una relación directa con las retribuciones hacia ésta, con un mayor compromiso, eficiencia y eficacia en las labores que se desempeñan.

Por ello, el talento humano no debe ser considerado un recurso más en la empresa. La nueva forma de ver el trabajo debe estar entonces bajo una perspectiva psicológica y humana. Su gestión adecuada en las empresas se convierte en una tarea prioritaria e impostergable. Las organizaciones deben buscar que su crecimiento sea permanente, producto del compromiso y la motivación generada en sus colaboradores, creando medios para que sean felices con lo que hacen, para que se sientan cómodos y seguros en el ambiente en el que se desenvuelven, equilibrados en su vida laboral y personal, satisfechos con el adecuado trato y con las compensaciones justas por su trabajo. 


\subsection{RSC y creatividad}

Son pocos los estudios realizados que relacionen la buena gestión de los stakeholders con la RSC (O'Riordan y Fairbrass, 2014) debido al constante cambio existente en las organizaciones fruto de la globalización económica. Hoy en día solamente las empresas más innovadoras, creativas y acumuladoras de capital son las que prevalecen en el tiempo (Popescu y Crenicean, 2012). En una sociedad basada en el conocimiento y la creatividad, las empresas han de ser flexibles para adaptarse a los cambios, siendo el capital intelectual la clave para sobrevivir con éxito en entornos altamente cambiantes (Parpandel, 2013). Si la organización desea competir y sobrevivir en entornos hostiles ha de fidelizar a su capital intelectual más valioso. Un capital intelectual que va evolucionando en el tiempo para adaptarse a nuevos retos, así como para ampliar sus conocimientos, tanto tácito como explícito, para luego aplicarlo en la innovación. Este proceso crea ventajas competitivas para la empresa, la cual se ve favorecida en entornos globales, sobre todo cuando encuentra océanos azules y verdes (Colvin y Saiz-Alvarez, 2015) caracterizados por el descubrimiento de nuevas oportunidades de negocio, así como por el fortalecimiento constante de su estructura productiva y comercial gracias al intelecto, la intuición y la creatividad.

Las organizaciones deberían utilizar todos sus recursos tangibles e intangibles para asegurarse el éxito en su supervivencia y desarrollo. De esta manera, los stakeholders irán construyendo una sociedad del conocimiento caracterizada por la transmisión de ingentes flujos de información en tiempo real por todo el planeta (Parpandel, 2013). Flujos de información que, para ser correctamente asimilados y en su caso mejorados, han de apoyarse en la educación. Una educación, a su vez, definida por su especialización y por su carácter innovador. Solo así será posible dinamizar unas organizaciones que, lejos de anquilosarse, vayan entrando cada vez más en nuevos entornos empresariales, tanto productivos como mercantiles, junto a una creciente especialización.

En términos de crecimiento regional, y cuando viene acompañada por una correcta política pública generadora de efectos crowding in, dicha especialización lleva hacia una diferenciación y posterior singularización de los productos, así como por la creación de valor dentro de la empresa, lo que influye a su vez en las regiones. De hecho, la especialización económica regional depende de la continua interacción entre la potencialidad de los mercados regionales para generar EBITDA para la empresa y el beneficio realmente obtenido (Karlsson y Johansson, 2011).

Dentro de la sociedad del conocimiento, es fundamental el papel del sector servicios, y en particular, a los servicios empresariales intensivos en conocimiento, también conocidos como KIBS (del inglés, Knowledge Intensive Business Services), que son considerados como los puentes de la innovación, la competitividad y el desarrollo, sobre todo cuando la innovación es grupal y no individual. De hecho, las KIBS permiten extraer más valor a las estrategias productivas y comerciales (Dell'Era, Landoni y Verganti, 2015). 
Dada las tendencias de consumo actuales existentes en el mundo, los consumidores de bienes y servicios aumentan las exigencias en términos de RSC y de alta calidad, y que de forma paralela sean empresas responsables con sus empleados, en donde no haya explotación laboral, se prevengan accidentes laborales, se genere mayor empleo con salarios justos y se cuide el medio ambiente en el desarrollo de su actividad productiva. Además, los consumidores valorarán más la calidad y fiabilidad de la información que les brindan las empresas respecto a los productos que les ofrecen. Según Vargas (2006), el consumidor manifiesta mayor poder en un entorno de globalización de mercado, en el cual sus exigencias se vuelven determinantes en el desarrollo empresarial. Lo cual no hay que descuidar.

\subsection{RSC y medio ambiente}

Es importante destacar y aceptar el entorno real en el que todos los agentes se desenvuelven, dicho entorno muestra una evidente interrelación existente entre las comunidades naturales y humanas. Esta relación se ve afectada a causa del desarrollo de actividades económicas sin una adecuada gestión ambiental por parte de la humanidad, generando desequilibrios. Así, restablecer el equilibrio entre el hombre y ambiente es también un importante desafío de los empresarios. Sin duda no se puede dejar de producir, pero hay que hacerlo de forma sostenible. Para ello se debe trabajar en el desarrollo de alternativas para la generación de ingresos, el uso de tecnología limpia, la utilización racional de la energía y el desarrollo de medios para el uso de la energía renovable. La implementación de un Sistema de Gestión Medio Ambiental (SGMA) supone una revisión de todos los procesos productivos de la empresa realizando los correspondientes cambios que comporten disminuciones en el consumo de agua, energía y materias primas o minimicen la producción de residuos y/o emisiones, lo cual significará una optimización de los costes de producción en general, a la vez que se cuida el ambiente (Azqueta, 2007).

Usando una muestra de 19 países en desarrollo durante un período de seis años, Boulouta y Pitelis (2014) sugieren que la RSC contribuye de forma especialmente positiva en aumentar tanto la competitividad de las empresas como el nivel de vida de la población, especialmente en aquellas naciones con bajos niveles de innovación. Esto es debido a que la RSC en combinación con procesos de innovación lleva a una redistribución eficiente de recursos y beneficio empresarial hacia el desarrollo de la comunidad en los países emergentes, de forma que se provocan externalidades positivas que benefician a toda la sociedad en su conjunto (Mukherjee y Ramani, 2011).

\subsection{RSC e innovación}

Sin duda, y para lograr cambios y una mayor competitividad, la innovación debe estar en el centro de la estrategia empresarial. De hecho, la concepción y puesta en marcha de políticas de buen gobierno, el imperio de la ley y los derechos de propiedad son esenciales para la eficiencia y la innovación (Porter y Kramer, 2006) al lograr un mayor grado de seguridad jurídica, lo que beneficia los procesos de inversión, sobre todo de capitales provenientes del exterior, y en última instancia a la competitividad de la empresa. De hecho, para lograr mayor competitividad la innovación se constituye en un elemento fundamental (Porter, 2011). 
La innovación afecta de forma especial a la RSC debido a que la innovación no debe entenderse como aquélla que únicamente afecta a los cambios tecnológicos, de acuerdo a Hill y Jones (2005), sino que una empresa innova en su manera de tomar decisiones, en el cambio de sus estructuras organizacionales, en su forma de relacionarse con los clientes y proveedores, en las características de desarrollo del negocio, en la estrategia de venta y en las políticas de marketing realizadas para la distribución, tanto minorista como mayorista, de sus bienes y servicios. Como resultado, el proceso de innovación es múltiple, lo que afecta a la corporación en su conjunto.

No se trata solo de conseguir unos mayores volúmenes de inversión en maquinaria y equipos, sino en lograr también modificaciones en los comportamientos, actitudes y habilidades. Se trata de aportar al desarrollo de recursos y capacidades de la empresa, a través de la innovación para generar mayor valor a partir de las actividades que en ella se desarrollan y, a la vez, generar beneficio a la sociedad en su conjunto. Como resultado, la innovación impacta sobre la RSC, debido a que mediante la innovación se logra excelentes resultados con valor agregado a las empresas a partir de la puesta en marcha de nuevas ideas. Es atreverse, arriesgarse a realizar acciones que convierten a sus empresas en pioneras, lo que mejora la imagen corporativa y de marca, y maximiza el bienestar social en su conjunto.

Por ello, ser una empresa innovadora requiere crear una cultura de continuos cambios en todas sus áreas. Necesita el compromiso de todos los grupos de interés (stakeholders) involucrados en la organización evitando la existencia de grupos de presión (lobbies). Una empresa innovadora con sensibilidad social a partir de la puesta en marcha de políticas basadas en RSC se caracteriza por su elevado grado de flexibilidad, su capacidad de ajuste ante cualquier imprevisto, su apertura hacia nuevos mercados, ideas y estrategias, y su amplia y efectiva política de comunicación y participación para poder capturar y explotar las ideas con el mayor éxito empresarial y social posible.

Como resultado, los beneficios de innovar no son ocultos. Por ejemplo, si una empresa mantiene una relación directa y coordinada con sus proveedores se asegura la calidad de la materia prima, el producto final, y las cantidades y tiempos requeridos. Todos ganan (estrategia win-win), tanto productor como proveedor y, por supuesto, el cliente recibe productos y servicios con una mayor calidad.

Así como dicha innovación, que no requiere de mayor inversión, sino de una actuación estratégica concreta, se pueden crear y ejecutar acciones que aún no han sido descubiertas 0 aún no han sido ejecutadas por falta de decisión. En el entorno de competencia en que se desenvuelven actualmente las empresas, sumado al desarrollo continuo y dinámico del conocimiento, es fundamental que la empresa sea innovadora para sobrevivir. En otras palabras, una empresa sin innovación va camino a su desaparición. Como resultado de todo ello, las empresas que desean sobrevivir, ser sostenibles y exitosas en el actual entorno dinámico deben innovar y, en ese sentido, la RSC es una variable estratégica y necesaria que deben gestionar unas organizaciones que están comprometidas con el desarrollo sostenible (Vargas, 2006). 


\section{3.- Método y análisis de resultados}

El objetivo de esta investigación es la exploración respecto a las innovaciones estratégicas en empresas de Guayaquil en el contexto de la RSC. Para ello se utilizó el método descriptivo siendo las unidades de investigación las empresas de Guayaquil (Ecuador). Se utilizó como técnica de recogida de información primaria la encuesta estructurada en cuatro secciones ([1] Datos generales, [2] El compromiso de la organización con la RSC, [3] Las acciones específicas de RSC que las empresas implementan con cada uno de los stakeholders y [4] Los beneficios percibidos por los empresarios desde la aplicación de la RSC), trabajándose con una muestra de 70 empresas siguiendo un muestreo no probabilístico, y de tipo secundaria, la revisión bibliográfica. Al ser el universo finito $(\mathrm{N}=1010)$ la fórmula utilizada para el cálculo del tamaño de la muestra para un error muestral (precisión) del $5 \%$ y un nivel de confianza (seguridad) del $95 \%$ es la siguiente:

$$
n=\frac{N \times Z_{\alpha}^{2} \times p \times q}{d^{2}(N-1)+Z_{\alpha}^{2} \times p \times q}=\frac{1010 \times 1,96^{2} \times 0,05 \times 0,95}{0,05^{2}(1010-1)+1,96^{2} \times 0,05 \times 0,95}=\frac{184,30076}{2,704976}=68,134 \simeq 68
$$

Se obtuvo la percepción de empresarios respecto a la conceptualización de la RSC y su relación con los stakeholders, la formalización en la implementación de la RSC en las empresas, las actuaciones en beneficio de los stakeholders, las acciones de protección ambiental y la identificación de los beneficios potenciales desde la RSC. Los encuestados respondieron personalmente, y no por vía telefónica ni telemática al cuestionario, sin que tuviesen que aportar evidencias de las respuestas, siendo la persona que contestó el máximo responsable de la organización.

Los resultados revelan el interés de las empresas guayaquileñas por innovar en sus estrategias implementando acciones que implican RSE. Si bien ello significaría implementar acciones integradas en sus diversos ámbitos como son: talento humano, gobierno, proveedores, sociedad y ambiente, se destaca en el estudio que las actuaciones de responsabilidad social en las empresas son aisladas. Integrarlas requiere un mayor esfuerzo, inversión y tiempo, pero más aún de compromiso y voluntad para concretarlo. Se está en el camino de la responsabilidad social, sin duda se avanza. Sin embargo, pocas son las organizaciones que de manera integrada intentan el esquema de RSC. Cabe enfatizar que la información presentada refleja la percepción de los empresarios.

Para los empresarios guayaquileños la RSC es tanto contribuir con el bienestar de la sociedad como orientar hacia la mejora de la calidad de vida de los profesionales de la empresa, mientras que únicamente en tercer lugar se sitúa el cuidado hacia el medio ambiente. Según se observa en el cuadro 1, un $83 \%$ de los empresarios asocia a la RSC con filantropía. 


\section{Cuadro 1. Conceptualización de la RSC para las empresas guayaquileñas}

\begin{tabular}{|lcc|}
\hline La RSC es... & SI & NO \\
\hline ... filantropía & $83 \%$ & $17 \%$ \\
... la contribución al bienestar de la sociedad & $63 \%$ & $37 \%$ \\
... la mejora de la calidad de vida del empleado & $52 \%$ & $48 \%$ \\
... el cuidado hacia el medio ambiente & $47 \%$ & $53 \%$ \\
\hline
\end{tabular}

FUENTE: Elaboración propia.

\section{1. ¿La RSC es parte de la filosofía de la empresa?}

Para abordar este interrogante se consideraron dos variables: [1] La inserción de la RSC en los principios de la empresa; y [2] la difusión y sociabilización al interior de la empresa. En cuanto a la declaración de RSC en su filosofía, el 77,14\% de las empresas respondieron afirmativamente, y en cuanto a la difusión, el 73\% indicaron que realizaban dicha comunicación al interior de sus empresas respecto de su compromiso con la RSC. Como se muestra, la mayoría de las empresas desde su percepción tendrían formalmente establecidas en su filosofía el compromiso de actuar con RSC. Como resultado, las empresas guayaquileñas, de una u otra manera, manifiestan realizar acciones basadas en RSC, ya sea por realizar donaciones, entregar bonos de comida, participar en acciones sociales o trabajar con fundaciones y Organizaciones No Gubernamentales (ONG).

De acuerdo a lo señalado por los directivos, el 94,28\% de las empresas se gestionan con RSC. Esta información, en un sentido literal significa que las empresas guayaquileñas respetan y cuidan el medio ambiente, actúan a favor de sus clientes, son motivadoras y justas con sus empleados, realizan una activa labor social y cumplen con las leyes. Sin embargo, la realidad empresarial ecuatoriana es que las acciones son aisladas, ya que si una empresa realiza tareas de cuidado al ambiente, no necesariamente realiza labores sociales u otras acciones, por lo que la RSC implementada no es integrada.

\subsection{Acciones de RSC que efectúan las empresas de Guayaquil}

Ante el resultado de que más del $90 \%$ de los empresarios encuestados indican que en su organización se ejerce alguna acción de RSE, se considera importante destacar dichas acciones. Según se observa en el cuadro 1 , el $70 \%$ de los encuestados señalan que ejecutan acciones de responsabilidad social tendientes a favorecer a los clientes. Entre ellas se destacan la calidad del producto ofrecido, calidad del servicio, buen trato, garantías, variedad de productos innovadores, capacitación en el uso y cuidado de los productos, obsequios por compras, cumplimiento de normas ambientales para ofrecer productos amigables con el medio ambiente, búsqueda de medios para mejorar la satisfacción de los clientes y decir siempre la verdad, no engañar a los clientes. 


\section{Cuadro 2. Acciones de RSC en las empresas de Guayaquil}

\begin{tabular}{|lcc|}
\hline Acciones RSC... & SI & N0 \\
\hline En las empresas & $90 \%$ & $10 \%$ \\
Con los empleados & $81,42 \%$ & $18,58 \%$ \\
Con el medio ambiente & $80 \%$ & $20 \%$ \\
Con los clientes & $70 \%$ & $30 \%$ \\
Con la sociedad & $50 \%$ & $50 \%$ \\
\hline
\end{tabular}

FUENTE: Elaboración propia.

El $81,42 \%$ de los encuestados indican que ejecutan acciones basadas en RSC tienden a favorecer a sus empleados, entre las que se destacan fundamentalmente la estabilidad laboral de los empleados con el cumplimiento de todos los derechos laborales, incluyéndose el acceso a la Seguridad Social, el fortalecimiento de la seguridad industrial en el puesto de trabajo, el pago de unas remuneraciones justas, el desarrollo de un adecuado ambiente de trabajo, el acceso a procesos de educación continua, la inclusión de incentivos por rendimiento (viajes, premios) y el también acceso a seguro privado, entre otros factores.

Por otra parte, el $80 \%$ de los encuestados también aseguran que son responsables para proteger y preservar el medio ambiente. Se destacan entre las labores amigables con el ambiente: el reciclaje de productos, la correcta captación, saneamiento y tratamiento del agua, la construcción de áreas verdes sobre todo en grandes ciudades, la oferta de productos amigables con el medio ambiente preservadores de la capa de ozono, el cumplimiento exhaustivo de normas ambientales locales, regionales y nacionales existentes en materia medioambiental, la siembra de arbustos y árboles, la realización de campañas informativas y la capacitación de especialistas en el cuidado del medio ambiente para la realización de estudios de impacto medioambiental. Las empresas indican trabajar en la obtención de certificaciones ambientales, el desarrollo de procesos tecnológicamente limpios, la adquisición de tecnologías amigables con el medio ambiente, el uso de plásticos biodegradables así como su reciclado, el control del ruido para evitar la contaminación acústica y el cumplimiento con las normas básicas de higiene para prevenir enfermedades.

Junto a todo ello, el $50 \%$ de los encuestados señalan que son responsables con la sociedad en general y que participan en obras de grupos sociales. Entre estas gestiones destacan: [1] El trabajo colaborativo de las empresas con diversas fundaciones; [2] El desarrollo de programas comunitarios de manera particular; [3] Los regalos a directivos y profesionales en Navidad; [4] La creación de equipos médicos; y [5] La capacitación, las donaciones y el trabajo en conjunto con instituciones locales, regionales y nacionales para favorecer a la sociedad en general. Tal como se presentan los resultados de percepción de los empresarios, parecería que no hay nada que hacer en el campo de la RSC en Ecuador. Sin embargo, el trabajo apenas ha comenzado, por lo que las empresas tienen enormes 
desafíos en este ámbito, tal y como se muestra en el cuadro 2, en donde el $87,1 \%$ de las empresas afirman tener beneficios por actuar con políticas basadas en RSC. A pesar de ello, el 61,4\% indica que uno de los mayores beneficios es el reconocimiento de la empresa por parte de los clientes, 10 que fortalece tanto la imagen corporativa como la marca, mientras que el 58,5\% afirma que la RSC aumenta la lealtad y la confianza de sus empleados con los beneficios que ello implica para la organización.

\section{Cuadro 3. Beneficios de la RSC percibidos en las empresas de Guayaquil}

\begin{tabular}{|lcc|}
\hline & SI & NO \\
\hline Se tienen beneficios por actuaciones basadas en RSC & $87,10 \%$ & $12,90 \%$ \\
Reconocimiento de los clientes & $61,40 \%$ & $38,60 \%$ \\
Aumenta la lealtad y la confianza del cliente & $58,50 \%$ & $41,50 \%$ \\
Mejora el ambiente laboral & $55,70 \%$ & $44,30 \%$ \\
Aumenta la productividad & $42,80 \%$ & $57,20 \%$ \\
Disminuye costes & $25,70 \%$ & $74,30 \%$ \\
Otros & $8,57 \%$ & $91,43 \%$ \\
\hline
\end{tabular}

FUENTE: Elaboración propia.

Por último, el $55,7 \%$ señala que se mejora el ambiente laboral, lo cual debiera aportar hacia una mayor productividad. Sin embargo, cuando se les pregunta sobre el impacto que la RSC tiene en la productividad, únicamente el $42,8 \%$ de los empresarios encuestados indican que hay un aumento en la productividad sin brindar información de medición donde se evidencie que la mayor productividad se debe a las acciones de RSC. De igual manera, ocurre con el impacto en el coste, en donde solo el $25,7 \%$ de los encuestados señalan que hay una disminución en los costes debido a la aplicación de políticas basadas en RSC. Respecto a estos dos últimos indicadores se ratifica el hecho de que las empresas no han implementado medios formales de medición integrada de los resultados de su gestión con RSC ya que, por ejemplo, no existen indicadores universales y comparables que midan el nivel de satisfacción de los empleados en cuanto a su motivación y la relación con acciones de RSC, lo que hace que toda la información respondida en la encuesta en relación con dicho tema tenga un carácter subjetivo. A esto se une el que algunas empresas ecuatorianas no disponen de sistemas contables aceptados universalmente en donde se incluyan los costes y los beneficios derivados de una buena gestión ambiental de las empresas, por lo que falta el diseño y la implementación de los modelos de gestión y el seguimiento en esta área. Unos sistemas de información integrada que permitan una toma de decisiones consecuente con los objetivos que se buscan alcanzar con el manejo socialmente responsable en las empresas. 
Por los beneficios que se perciben en el cuadro 2, en donde el $94 \%$ de las empresas expresan que es necesario gestionar con RSC en sus organizaciones para mejorar la imagen de la empresa, involucrar más a los empleados con la actividad central de la organización y lograr un mayor cuidado del medio ambiente, según se refleja en el cuadro 3, es necesario seguir la introducción en las empresas ecuatorianas, y en concreto en las guayaquileñas, políticas basadas en RSC para que así se contribuya a mantener un buen posicionamiento en el mercado de forma sostenida y sostenible, lo que es beneficioso para la organización en su conjunto.

\section{Cuadro 3. ¿Por qué es necesaria la gestión con RSC?}

\begin{tabular}{|lcc|}
\hline & SI & NO \\
\hline Mayor cuidado del medio ambiente & $57,14 \%$ & $42,86 \%$ \\
Mejorar la imagen & $52,80 \%$ & $47,20 \%$ \\
Involucrar más a los empleados & $51,42 \%$ & $48,58 \%$ \\
\hline
\end{tabular}

FUENTE: Elaboración propia.

Por último, y en cuanto a los profesionales (directivos y empleados) que trabajan en la organización, es relevante involucrarlos en el proceso, ya que se trata no de un empleo en el que el colaborador solamente cumple horarios y actividades, sino que se genera un compromiso. Así, el profesional se termina preocupando por el resultado no solo individual de su trabajo, sino también organizacional. A pesar de este hecho altamente beneficioso para la empresa, para casi la mitad de los empresarios encuestados la RSC no es necesaria para involucrar y comprometer a los empleados con los objetivos de la empresa.

\section{4.- Conclusiones}

Las empresas ecuatorianas en Guayaquil implementan de forma aislada acciones de RSE, pero de forma no integrada, ya que son muy pocas las organizaciones, generalmente extranjeras, las que manejan e implementan acciones integradas de RSC. A pesar de este hecho, que pueda parecer negativo a primera vista, lo interesante es que existe la preocupación y el interés por parte de las empresas ecuatorianas para implementar estrategias dentro de este ámbito. Para el empresariado ecuatoriano, y en concreto el guayaquileño, la RSC es considerada como un medio para lograr ventajas competitivas, porque está generalmente extendida la idea de si se descuida la atención a las necesidades del talento humano y la sociedad y no se protege al medio ambiente, las empresas no tendrán el aval social para mantenerse en el mercado. Por ello, la RSC incita a los empresarios a no 
enfocarse únicamente en ser más competitivos, sino que, además, los stakeholders tienen mayor poder e incidencia sobre ellos, sobre todo aquellos que más se ven beneficiados por la aplicación de las estrategias basadas en RSC dentro de las organizaciones.

Por otra parte, destaca en Ecuador que las empresas guayaquileñas manejan definiciones diversas de la RSC, aunque la mayoría coinciden en que RSC sea contribuir al bienestar de la sociedad, la diferencia se da en las formas de actuación que cada una adopta bajo este enfoque. Al resumir las actuaciones de las empresas con cada stakeholder, se resalta la percepción de los empresarios en relación al talento humano, ya que aseguran cumplir con las exigencias de las leyes laborales en cuanto a Seguridad Social y el pago de salarios justos según lo establecido en los convenios laborales, además de brindar incentivos, formación y crecimiento a sus colaboradores.

Sin embargo, no solo los adecuados y justos niveles de salarios son de interés para los empleados. También requieren tener un adecuado espacio y un buen ambiente de trabajo y seguridad en la labor que realizan. En caso de no tenerlo, buscarán nuevas oportunidades de crecimiento profesional y personal, al querer tener armonía con ellos mismos y con su entorno, además de disponer de un correcto equilibrio entre su actividad laboral, familiar y personal buscando la conciliación laboral. Sin embargo, en este aspecto, aún hay mucho trabajo pendiente por realizar en Ecuador.

En cuanto al medio ambiente, las empresas cumplen parcialmente de un sistema de gestión de cuidado ambiental y pocas empresas tienen certificaciones ambientales. Sin embargo, se muestra interés y esfuerzo de trabajo para mejorar en este sentido, sobre todo por parte de las empresas transformadoras. Si bien aún persisten los problemas y los efectos negativos en materia medioambiental desde la actividad productiva, se evidencia el inicio de un recorrido por el camino hacia la producción con sostenibilidad ambiental.

En relación a los consumidores, es un aspecto que ha sido más descuidado en relación a la RSC. Esto es así porque los consumidores no son muy exigentes a la hora de exigir información sobre la composición y la trazabilidad de los productos en el momento de ejercer su compra. Por ello, el Ministerio Ecuatoriano de Industrias y Productividad se encuentra actualmente realizando una campaña de concientización del consumidor, creando espacios para que se pueda informar y los consumidores puedan manifestar sus opiniones respecto a lo que encuentra en el mercado e incluso demandar situaciones que les afecten. Sin embargo, todo ello apenas ahora está iniciando, por lo que no es comparable la situación de protección al consumidor que existe en los países más avanzados del mundo.

En cuanto a los beneficios declarados por los empresarios, van desde la mejor calidad de vida de los empleados y la mayor satisfacción de los clientes, hasta el aumento de la competitividad de la empresa. En este aspecto cabe resaltar que uno de los grandes beneficios que se percibe es mejorar la imagen corporativa de la organización, lo que constituye un valor intangible y una fuente importante de generación de ventaja competitiva, tanto de primer orden o de rango superior, en el caso de la inno- 
vación, como de segundo orden o de rango inferior, si la empresa compite con bajos costes laborales unitarios.

El estudio muestra que hay muchas actuaciones que son consideradas como RSC y se presentan claras intenciones de actuar con dicho enfoque. Sin embargo, hay mucho camino por recorrer, sobre todo en torno a la implementación estratégica integrada de este modelo de gestión en las empresas de Guayaquil. Resultado de este bajo grado de implementación de la RSC en las empresas guayaquileñas, algunas organizaciones se ven afectadas por la generación de un bajo valor agregado, hay inestabilidad laboral por continuos cambios de empleados por su desmotivación, baja productividad y pérdida de clientes ante productos de una calidad no adecuada a las exigencias de los mismos. La menor disponibilidad de recursos y el no aval de la sociedad, lleva a medio plazo hacia el claro destino de la desaparición del mercado.

Por ello, y dada la importancia y las ventajas conseguidas con la aplicación de la RSC en la empresa, abogamos por la extensión y la profundización de dichas buenas prácticas empresariales para beneficiar a la empresa ecuatoriana. De esta manera se podrá conocer mejor el impacto medioambiental de las empresas, así como generar información que permita valorar las aportaciones que realizan las empresas a la sociedad y a la economía en general con la implementación de este tipo de gestión. A la vez, que dejen visualizar las acciones y las políticas específicas que se requieren para contribuir al desarrollo del buen vivir en Ecuador.

\section{Bibliografía}

AZQUETA, D. (2007): Introducción a la economía ambiental, McGraw Hill Interamericana, México.

BERNAL, C. (2010): Metodología de la investigación. Administración, Economía, Humanidades y Ciencias Sociales, Tercera Edición, Colombia: Pearson Educación.

BOCQUET, R. \& MOTHE, C. (2011): "Exploring the relationship between CSR and innovation: A comparison between small and largesized French companies", La Revue des Sciences de Gestion, 80, 101-119, http://www.larsg.fr.

BOULOUTA, I. \& PITELIS, C. (2014): "Who Needs CSR? The Impact of Corporate Social Responsibility on National Competitiveness", Journal of Business Ethics, 119(3), 349-364, http://www.springer.com/social+sciences/applied+ethics/journal/10551. 
COLVIN, J. \& SÁlZ-ÁLVAREZ, J.M. (2015): "Purple Oceans and the Mergers \& Acquisitions Virtuous Circle. New Concepts for the Theory of Mergers \& Acquisitions". En: Tavidze, A., Progress in Economics Research, 32, Nueva York (EE.UU.): Nova Press, https://www.novapublishers.com/catalog/product_info.php?products_id=54773\&osCsid=c998e547e0a70b4f1bb138f0a23cffa6.

DELL'ERA, C., LANDONI, P. \& VERGANTI, R. (2015): "From Creative Individuals to Creative Capital: Value Creation and Appropriation Strategies of Creative Knowledge-intensive Business Services", International Journal of Innovation Management, 19(2), 1-24, http://www.worldscientific.com/doi/pdf/10.1142/S1363919615500164.

EPSTEIN, M. (2009): Sostenibilidad empresarial. Administración y medición de los impactos sociales, ambientales y económicos, Eco Ediciones, Colombia.

HILL, Ch. \& GARETH, J. (2012): Administración Estratégica: Un Enfoque Integrado, McGraw-Hill, México.

INFORME FORÉTICA (2011): Evolución de la Responsabilidad Social de las Empresas en España, Madrid, España.

INFORME SOBRE DESARROLLO HUMANO (IDH) (2013): Sostenibilidad y Equidad: Un mejor futuro para todos Programa de las Naciones Unidas para el Desarrollo (PNUD), Nueva York.

INFORMES INEC (2014): Coeficiente de Gini, marzo.

KARLSSON, C. \& JOHANSSON, B. (2011): "Knowledge, Creativity, and Regional Development", Working Paper Series in Economics and Institutions of Innovation, 148, Royal Institute of Technology \& CESIS, Suecia.

MACGREGOR, S.P. \& FONTRODONA, J. (2008): "Exploring the Fit between CSR and Innovation", IESE Research Papers, D/759, Universidad de Navarra, Pamplona, España.

MUKHERJEE, V. \& RAMANI, S.V. (2011): "Voluntary agreements and community development as CSR in innovation strategies", MERIT Working Papers, 16, United Nations University.

NORMAS ISO 26000 (2010): Guía de Responsabilidad Social, Washington.

O'RIORDAN, L. \& FAIRBRASS, J. (2014): "Managing CSR Stakeholder Engagement: A New Conceptual Work", Journal of Business Ethics, 125(1), 121-145, http://www.springer.com/social+sciences/applied+ethics/journal/10551.

OGLIASTRI, E., PEINADO, E. \& IMRE, P. (2007): El argumento empresarial de la RSE: 9 casos de América Latina y El Caribe, INCAE, Costa Rica.

OLCESE, A., RODRíGUEZ, M. \& ALFARO, J. (2008): Manual de la Empresa Responsable y Sostenible, Mc Graw Hill, Madrid, España. 
PARPANDEL, D.E. (2013): "The Role of Intellectual Capital in a Knowledge-based Society", Management Strategies Journal, $47-52$, http://onlinelibrary.wiley.com/journal/10.1002/(ISSN)1097-0266.

PEINADO, E. (2011): La RSC en América Latina. Manual de gestión. La Responsabilidad Social de la Empresa en América Latina, FOMIN-BID, Fondo Multilateral de Inversiones-BID, Computere.

PELEKAIS, C. \& AGUIRRE, R. (2008): Hacia una Cultura de Responsabilidad Social, Pearson, México.

PERRINE, F. (2013): "The Complementarity of Corporate Social Responsibility and Innovation: Evidence from Belgian Firms", Global Journal of Business Research, 7(5), 99-113, http://www.theibfr.com/gjbr.htm.

POPESCU, M. \& CRENICEAN, L.C. (2012): "Considerations Regarding Improving Business Competitiveness from an Entrepreneurial Perspective", International Journal of Academic Research in Accounting, Finance and Management Sciences, 2(1), 9-15.

PORTER, M. (1999): Ventaja competitiva: Creación y sostenibilidad de un desempeño superior, Compañía Editorial Continental, México.

PORTER, M. \& KRAMER, M. (2006): "Estrategia y sociedad. El vínculo entre ventaja competitiva y responsabilidad social corporativa", Harvard Business Review, 21, 78-91.

SAIZ-ÁLVAREZ, J.M. (2008): "Empresa familiar y capital intelectual: una visión desde el metaconocimiento", en Saiz-Álvarez, J.M. \& Gámez, J. (Eds.) (2014): La empresa familiar ante la crisis. Nuevas ideas, nuevas estrategias, Bogotá (Colombia): Ediciones de la Universidad de La Salle, 9-29.

SCHWALB, M. (2011): "La responsabilidad de la empresa ante los consumidores". En: Manual de gestión. La Responsabilidad social de la Empresa en América Latina, FOMIN-BID, Computere.

VACA, R., MORENO, M. \& RIQUEL, F. (2007): "Análisis de la Responsabilidad Social corporativa desde tres enfoques: stakeholders, capital intelectual y teoría institucional". En: Ayala, J.C., Conocimiento, innovación y emprendedores: Camino al futuro, Universidad de La Rioja, 31303143.

VARGAS, J. (2006): Responsabilidad Social Empresarial (RSE) desde la perspectiva de los consumidores, CEPAL-Publicación de las Naciones Unidas, Santiago de Chile. 
